\title{
Evolución de la conductividad en la Albufera de Valencia entre 1985 y 2018
}

\author{
Juan Soria*, Susana Romo, Lucía Vera-Herrera, Sara Calvo, Xavier Sòria-Perpinyà \\ y Javier Pérez \\ Instituto Cavanilles de Biodiversidad y Biología Evolutiva. Universitat de València. 46980-Paterna. \\ * Corresponding author: juan.soria@uv.es
}

Received: 27/11/19

Accepted: 22/05/20

\section{RESUMEN}

Evolución de la conductividad en la Albufera de Valencia entre 1985 y 2018

La laguna costera de la Albufera de Valencia es una masa de agua oligohalina rodeada de un antiguo marjal, transformado en cultivo del arroz desde hace más de 200 años. La presión de las aportaciones superficiales mantiene la conductividad en valores estables en torno a la media anual, modificada puntualmente al alza sólo en los periodos de sequía. La concentración de cloruros es la variable más relacionada con la conductividad. En el estudio de la serie temporal se observa que en el último decenio hay una tendencia significativa al aumento de los valores mínimos de la conductividad, lo cual indicaría una disminución de la cantidad de agua dulce que llega a la laguna. La medida de esa variable sería un buen indicador de las alteraciones que se pueden producir en un escenario previsible de cambio climático con descenso de las precipitaciones y de las aportaciones de agua dulce.

Palabras clave: laguna costera, salinidad, conductividad, serie larga

\section{ABSTRACT}

Changes of the conductivity in the Albufera of Valencia lagoon between 1985 and 2018

The Albufera of Valencia (Eastern Spain) is an oligohaline coastal lagoon, surrounded by an ancient marsh transformed into rice fields about 200 years ago. The surficial water inflows maintain the conductivity in steady values around the annual average, with occasional increases in periods of droughts. The concentration of chloride is the variable most related to conductivity. In this study of its long-term conductivity changes, we have observed that there has been a significant trend toward an increase of minimum values in the last decade, which could indicate a decrease in freshwater inputs to the lagoon. The measurement of this variable would be a good indicator of the alterations that may occur in a foreseeable scenario of climate change with a decrease in both rainfall and freshwater inflows.

Key words: lagoon, salinity, conductivity, long-term series 


\section{INTRODUCCIÓN}

La Albufera de Valencia es uno de los principales humedales a nivel nacional y de gran importancia en el Mediterráneo occidental. La laguna costera y el marjal circundante, junto con la restinga que la separa del mar, fueron declarados Parque Natural el 23 de julio de 1986 por su elevado valor ambiental, paisajístico y cultural, con el fin de frenar la degradación sufrida durante la década de los 70, debida a la presión urbanística, y asimismo recuperar sus valores originales. El Parque Natural ocupa 21120 hectáreas de superficie, de las cuales unas 3000 corresponden al lago y las aguas someras. A nivel internacional, en 1990 se incorporó a la Lista de Zonas Húmedas de Importancia Internacional de la Conferencia de Ramsar y en 1994 fue declarada Zona de Especial Protección de Aves, siendo por tanto Lugar de Interés Comunitario para la Directiva Hábitats de la Unión Europea (Soria, 2006).

La laguna es el resultado del cierre de un golfo marino por el crecimiento de una flecha arenosa de sedimentos fluviales que ha formado la actual restinga a partir de sucesivas alineaciones dunares cuyo inicio ocurrió hace unos 8000 años (Rosse1ló, 1972). El cierre total de la comunicación con el mar tuvo lugar hacia finales del siglo XVIII, momento en el cual los canales de intercomunicación se reducen en dimensiones a lo necesario para el desagüe de la laguna y se regula su nivel con la instalación de compuertas que limitan la circulación del agua. Simultáneamente, se ponen en cultivo los marjales próximos a la laguna para producir arroz, después de que el Duque de Híjar, Señor de Sollana (propietario de gran parte del territorio) ordenara construir la prolongación de la Acequia Real del Júcar (construida en 1270) desde el sifón del río Magro en Guadassuar hasta Albal, proporcionando así agua dulce para el cultivo de la zona (Peris, 1991). En ese momento se produjo con seguridad la mayor alteración ecológica del ecosistema de la Albufera, de ambiente marino abierto al mar hasta entonces, con aportaciones de agua dulce procedentes de los drenajes superficiales de su cuenca, a ambiente de agua dulce, que recibía continuamente las escorrentías agrícolas y poseía ya una barrera casi total a la entrada de agua marina y de las especies que de allí procedían. Este cambio quedó reflejado en el sedimento y fue estudiado por Margalef y Mir (1973) y, recientemente, en los trabajos de Marco-Barba (2010) y Rodrigo et al. (2010).

En general, la evolución de la salinidad en una laguna costera es el fruto de las interacciones entre la llegada de agua dulce desde la cuenca superficial, la entrada de agua marina y la evaporación. En los sistemas comunicados con el mar dependerá de si se comportan con circulación estuario-positiva, donde domina las aportaciones de agua dulce normalmente o estuario-negativa, cuyas entradas de agua marina son más importantes (Garrison, 2013). En el primer caso, el resultado es una laguna de aguas salobres, donde (si existe profundidad suficiente) puede haber un gradiente de densidad salino, de forma que las aguas más saladas se sitúen en las capas profundas; en los casos de mezcla debido a los temporales, se recupera la homogeneidad de la columna de agua. En el segundo caso, el resultado es una laguna de aguas hipersalinas, donde la entrada constante de agua marina y la evaporación, unida a la escasa aportación de aguas dulces, origina una concentración salina superior a la del agua marina. También puede existir estratificación salina si hay suficiente profundidad y estabilidad vertical. En ambos casos, el resultado es un ecosistema sometido a fluctuaciones de salinidad, tanto por causa de los temporales marinos como por los temporales de lluvias, en los cuales cambian súbitamente las condiciones de salinidad y, por lo tanto, encontraremos una biota adaptada a estas variaciones. Además, en un escenario de cambio climático, las condiciones ambientales de estas lagunas costeras pueden alterarse profundamente en función de las variaciones de la pluviometría o del aumento del nivel del mar (Le Bars et al., 2017). El descenso de la pluviometría lleva al sistema hacia estados hipersalinos, mientras que el ascenso del nivel del mar facilita la entrada de aguas marinas e, incluso, la desaparición de las restingas, con lo cual el ambiente se tornaría netamente marino.

En el caso concreto de la Albufera de Valencia, el cambio en la salinidad fue una consecuencia de la actividad humana en el territorio circundante, con la puesta en cultivo de las tierras que formaban parte del marjal y el aumento de las aportaciones de agua dulce a través de la Acequia 
Real del Júcar. Esta modificación de usos ha llevado a los estudiosos a hablar del cambio de la "Albufera de los pescadores" a la "Albufera de los agricultores", en la cual la gestión del agua se realiza en función de los usos agrícolas y la decadencia de la pesca relega su influencia en la gestión (Jégou \& Sanchis-Ibor, 2019).

A mediados del siglo XX comienza el desarrollo urbano e industrial en las poblaciones situadas al oeste de la Albufera y entre 1972 y 1974 tiene lugar la alteración del estado trófico de la laguna, lo cual genera la situación de laguna turbia hipertrófica que conocemos actualmente. Las causas están bien descritas en numerosos trabajos publicados desde aquellos años (Primo et al., 1975; Vicente \& Miracle, 1992; Romo et al., 2005; Romo et al., 2013; Soria et al., 2019).

Esa visión del lago en mal estado ecológico ya desde finales de los años 1970 contrastaba con el buen estado en la memoria de ciudadanos e investigadores unos años antes. Y comenzaron los primeros trabajos con el fin de evaluar los niveles de contaminación existentes y su estado ecológico realizados por la Universidad Politécnica de Valencia (Primo et al., 1975) y la Universidad de Valencia (Serra et al., 1984). Con la calificación como Parque Natural en 1986, se sucedieron los trabajos para conocer en profundidad el estado del ecosistema (redacción del Plan de Saneamiento), la ejecución de las primeras infraestructuras de recogida de aguas residuales (construcción del colector oeste), la depuración de aguas en las poblaciones de la cuenca (construcción de depuradoras de aguas residuales) y la implantación de otras medidas de mejora ambiental (construcción de filtros verdes), síntesis recogida documentalmente en la Tesis Doctoral de Mondría (2010). La Generalitat Valenciana asume el seguimiento de la calidad del agua de la laguna y desde 1991 existe una toma de datos regular de las aguas de la Albufera, así como otras medidas hidrológicas de interés, en las que también participa en ocasiones el Organismo de Cuenca (Confederación Hidrográfica del Júcar).

Recopilando las series de datos existentes desde los primeros estudios, el objetivo del presente trabajo es presentar y estudiar la serie temporal de los valores de conductividad, cuyos datos iniciales son discontinuos para 1974, 1980,

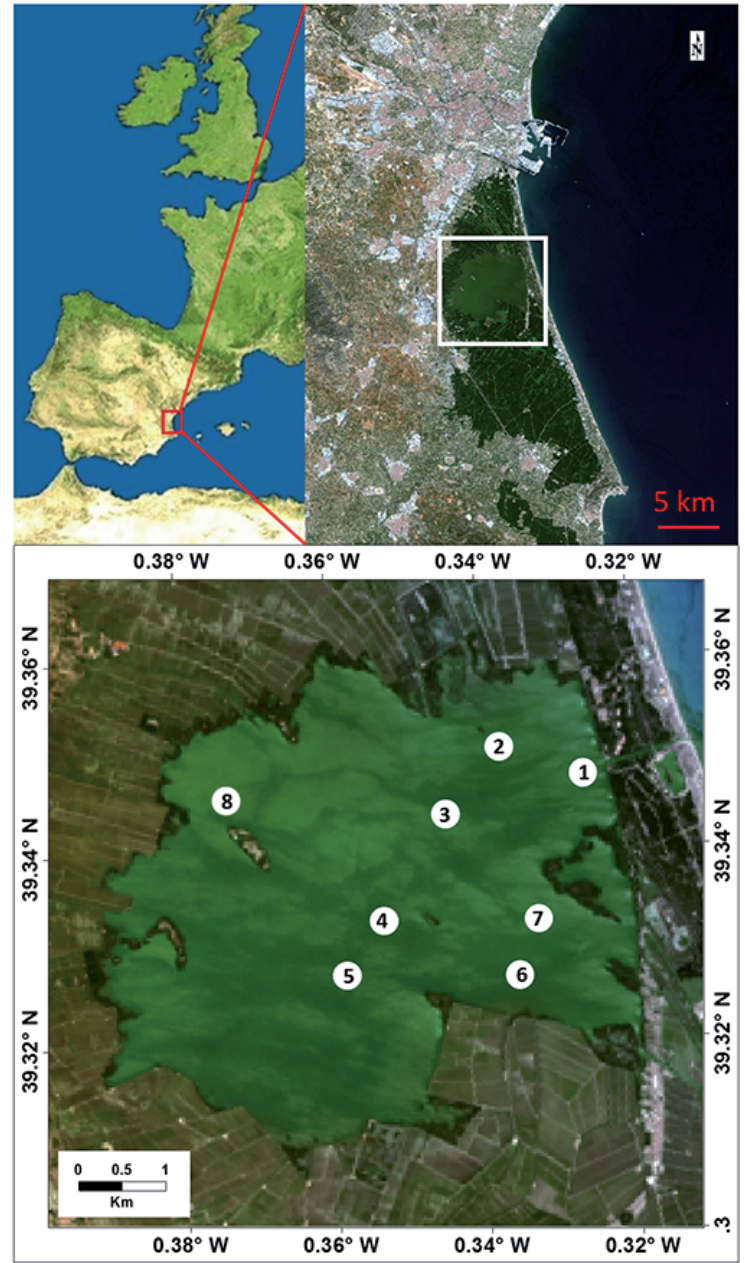

Figura 1. Ubicación de la Albufera de Valencia en la costa este de la Península Ibérica. Composición a partir de una imagen estival del satélite Sentinel-2, indicando los puntos habituales de toma de muestras (modificado de Soria-Perpinyà et al., 2020). Location of the Albufera de Valencia on the Eastern coast of the Iberian Peninsula. Composition from a summer image of the Sentinel-2 satellite, indicating sampling sites (modified from Soria-Perpinyà et al., 2020).

1985-1988 y con registro más continuo desde el verano de 1991 en adelante. A partir de ellos se estudia la serie temporal y las tendencias existentes en la misma.

\section{MÉTODOS}

La red de puntos de muestreo habitual en la Albufera está formada por ocho lugares que pretenden 
abarcar la heterogeneidad espacial que se puede presentar en la laguna debida a la diferente cantidad y calidad de las aportaciones que llegan por las acequias que allí desembocan. Son ocho puntos, de los cuales tres están ubicados, dos en el tercio central y tres en el tercio sur, cuya situación concreta se puede observar en la figura 1. No siempre se han tomado datos en los ocho puntos; en ocasiones se han tomado en tan solo cuatro de ellos, pero al menos siempre una muestra lo ha sido en el tercio norte, otra en el tercio sur y otra en la zona central. Las medidas fueron realizadas por los distintos autores de los trabajos revisados y también, desde 1991, por el personal al servicio de la gestión del Parque Natural. Las medidas de conductividad se realizaron directamente en el lago mediante un conductímetro previamente calibrado y referidas al estándar de $25^{\circ} \mathrm{C}$. A partir de las medidas individuales de la conductividad, se ha calculado una media para cada una de las zonas y, de los tres valores, se ha calculado el que luego se ha utilizado en la serie temporal para cada fecha de muestreo y resumido para cada año. El cloruro se determinó en sesenta muestras puntuales de los años 1985-1988 y 2016-2017 por el método de Möhr (Yoder, 1919), mediante la valoración de un volumen exacto de muestra de agua con nitrato de plata de concentración conocida, utilizando cromato potásico como indicador. Sobre los resultados se han realizado un análisis de los promedios según el t-test de Student, un análisis de homocedasticidad, una prueba de tendencias según Mann-Kendall y la prueba de Montecarlo de distribución aleatoria en torno a la media. Sobre la serie completa de datos y en partes de ella se ha realizado un análisis de autocorrelación para descubrir patrones en la serie de datos. El software estadístico utilizado ha sido PAST versión 3.26 (Hammer et al., 2001).

\section{RESULTADOS}

Se han recopilado los datos bibliográficos de 1974 (Primo et al., 1975) y 1975 (Del Río Y Fernández, 1980). Desde 1980 a 2018 los resultados proceden de las muestras estudiadas en 435 fechas, de las cuales se registraron ocho en 1979-1980 y las restantes, a lo largo del periodo entre 1985 y 2018 , con frecuencia aproximadamente mensual o incluso con dos muestreos en algunos meses. Además de la conductividad, se tomaron otras variables para conocer el estado trófico del lago (temperatura, oxígeno disuelto, clorofila $a$, nutrientes), cuyos resultados no se encuentran publicados, salvo los de algunos periodos parciales (Romo et al., 2005, 2013; Soria, 1997; Soria et al., 2001; Soria \& Vicente, 2002; Soria et al., 2005). La tabla 1 presenta un resumen de estos datos en el periodo 1980-1981 y en 2016-2018.
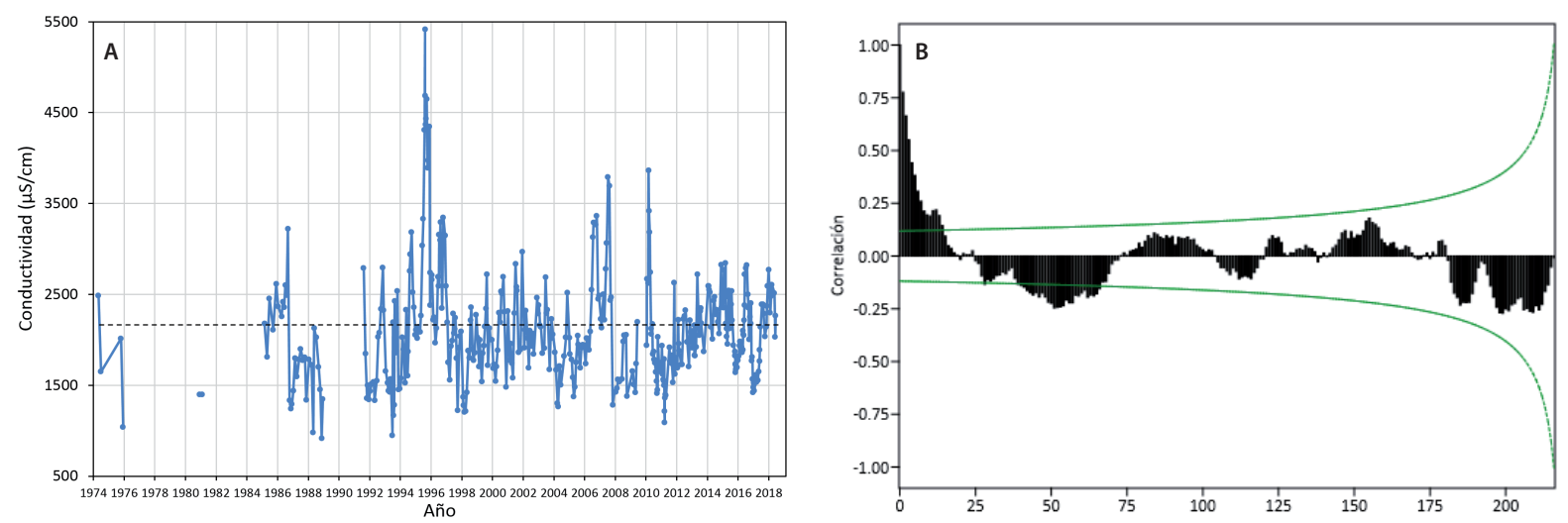

Figura 2. A. Dinámica de la conductividad en la Albufera de Valencia para el periodo de estudio (media espacial). La línea discontinua indica el valor promedio de la serie. B. Autocorrelación de la serie temporal, la línea verde indica el intervalo de confianza del $95 \%$. $A$. Dynamics of Albufera de Valencia conductivity for the study period (spatial average). The dashed line indicates the average value of the full series. B. Time series autocorrelation, the green line indicates the $95 \%$ confidence interval. 


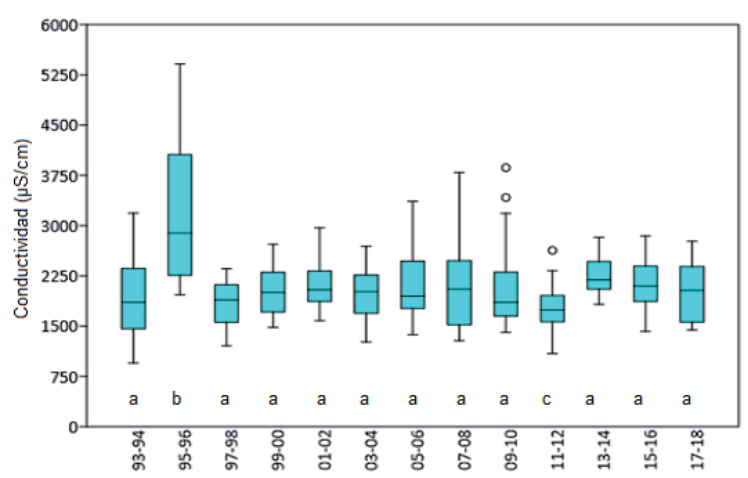

Figura 3. Gráfico de cajas de la serie temporal de la conductividad de la Albufera de Valencia, agrupada por bienios. Las letras indican los grupos que presentan diferencias estadísticamente significativas $(p<0.05)$. Boxplot of the time series of Albufera de Valencia conductivity, grouped by biennium. Letters indicate statistically significant $(\mathrm{p}<0.05)$ differences among groups.

El primer dato contrastado acerca de la salinidad en la Albufera de Valencia aparece en los trabajos de Zariquiey (1935) sobre los decápodos en la laguna, que fue citado por Pardo (1942). Se da el valor de $430 \mathrm{mg} / \mathrm{l}$ de cloruro sódico, que equivalen a $7.13 \mathrm{mM}$, cifra similar a las que tenemos en nuestra serie estudiada.

A lo largo del periodo estudiado, el análisis descriptivo presenta un valor promedio de la conductividad de $2103 \mu \mathrm{S} / \mathrm{cm}$ y una desviación estándar de $603 \mu \mathrm{S} / \mathrm{cm}$. En el sistema de clasificación de aguas salobres de Venecia, esto correspondería a aguas oligohalinas (ASLO, 1958). Las cifras más bajas de conductividad de la serie temporal fueron de $880 \mu \mathrm{S} / \mathrm{cm}$ en diciembre de 1975 y de $915 \mu \mathrm{S} / \mathrm{cm}$ en noviembre de 1988, justo en unos momentos tras lluvias intensas. En la parte opuesta, el valor más elevado correspondió a agosto de 1995, con $5414 \mu \mathrm{S} / \mathrm{cm}$. Debemos señalar que, así como los valores menores se presentaron en una fecha determinada ya indica$\mathrm{da}, \mathrm{los}$ once mayores valores de conductividad se dieron en el periodo entre el 3 de agosto y el 30 de noviembre de 1995 (Fig. 2A).

La representación de los valores agrupados por bienios (Fig. 3) muestra diferencias significativas de acuerdo con el t-test entre el bienio 1995-1996, que tiene los valores más elevados (grupo b, media: $3139 \mu \mathrm{S} / \mathrm{cm}$ ), y los demás

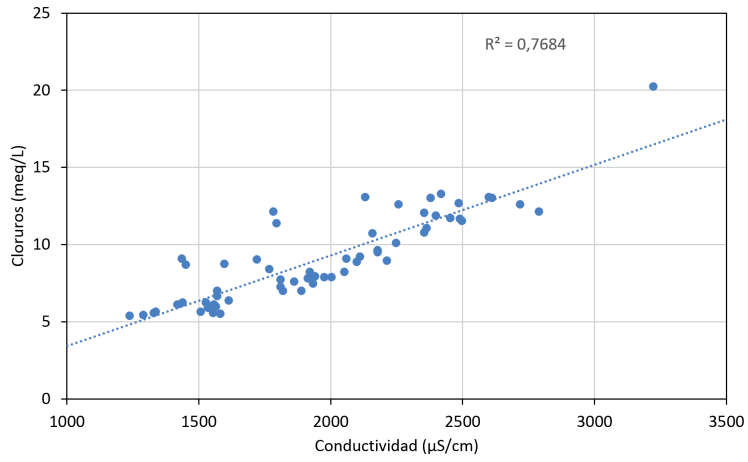

Figura 4. Relación entre la conductividad y la concentración de cloruros en un subconjunto de sesenta muestras de la serie temporal estudiada en la Albufera de Valencia, indicando el coeficiente de determinación de Pearson. Relationship between conductivity and chloride concentration in a subset of sixty samples of the Albufera de Valencia time series studied, indicating Pearson's determination coefficient.

(grupo a). Por otra parte, el bienio 2011-2012 presenta el conjunto de valores más bajos significativamente (grupo c, media: $1781 \mu \mathrm{S} / \mathrm{cm}$ ) respecto a los demás periodos.

A partir de los análisis químicos que se realizaron para conocer la concentración de cloruros del agua (Soria, 1997), se ha observado una relación positiva y significativa $(\mathrm{r}=0.877, \mathrm{n}=60$, $p<0.001$ ) entre la conductividad y dicha variable en las aguas (Fig. 4), como cabría esperar en un ecosistema lagunar costero influenciado por las aguas marinas. La tabla 1 muestra un resumen del valor de los cloruros en el periodo de estudio.

La prueba de Mann-Kendall para el estudio de tendencias indica que no existe una tendencia significativa a lo largo de la serie temporal, tanto para la serie muestral como para la serie anual ( $p=0.46$ y 0.34 , respectivamente). Sin embargo, si realizamos este estudio con los valores mínimos (Fig. 5), sí se observa una tendencia significativa creciente de la conductividad ( $p=0.0007)$. A pesar de ello, la prueba de Montecarlo de distribución de los valores muestrales de la conductividad en torno a la media sugiere que la distribución es totalmente al azar $(p<0.0001)$. La misma prueba con los datos anuales no es significativa e indica que la serie de las medias anuales no se distribuye al azar $(p=0.73)$. Por otra parte, se han explorado las tendencias de los valores mínimos 
de conductividad en los valores desde 2008 al presente, constatándose también una tendencia creciente significativa $(p=0.019)$.

La prueba de homocedasticidad de BreuschPagan resultó significativa $(p<0.0001)$ y muestra que, al igual que no existe una tendencia del valor de la conductividad en torno a la media, tampoco aumenta la dispersión significativamente.

Por último, el estudio de autocorrelación de los valores de conductividad entre 1985 y 2018 sugiere una distribución que tiende a la autocorrelación (Fig. 2B). Las oscilaciones aparecen de forma cíclica a lo largo del periodo de estudio, con un periodo de unos once años. Los valores más bajos se presentan en los periodos de lluvias importantes y los altos en los periodos de sequía (según los datos de AEMET, 2019), mostrando la relación entre la conductividad y las aportaciones de aguas dulces ya descritas en otras publicaciones (Soria et al., 2005).

\section{DISCUSIÓN}

En trabajos previos se estudió por separado en periodos concretos la conductividad en la Albufera de Valencia. En Soria et al. (2001) se comprobó la importancia de las avenidas como el factor que contribuye a disminuirla por aportación de escorrentía superficial y por la lluvia recogida en la cuenca. Por otra parte, en Soria et al. (2005) se estudió cómo la conductividad estaba relacionada con el regadío en el entorno a la laguna y, dado que la conductividad del agua del río Júcar está en torno a $1000 \mu \mathrm{S} / \mathrm{cm}$, cuanta más agua se suministre a la zona regable, más escorrentía llegará a la laguna y, por lo tanto, su conductividad descenderá. Al contrario, en los periodos de sequía, donde ni llegan escorrentía superficial ni sobrantes de riego, e incluso se aprovecha el agua de la laguna para el riego, se producen una salinización moderada y aumento de la conductividad, como se ha observado en el bienio 1995-1996. Este periodo coincidió con una sequía extraordinaria, en la cual la llegada de aguas al lago fue nula, hasta que ocurrió una tormenta de primeros de diciembre. Incluso durante el periodo de cultivo del arrozal se bombeó el agua desde el lago hacia los arrozales, lo cual favoreció la entrada de aguas marinas por filtración a través de la restinga y el consiguiente aumento de la conductividad. Tal y como sucede en otras lagunas costeras, la meteorología y los usos del territorio son los factores que más influyen en la mineralización de las aguas.

Por ello, salvo en los dos periodos indicados, la conductividad de la laguna permanece estable, con sus subidas y bajadas durante el año, fruto de las aportaciones, sin observarse una tendencia general al aumento de la conductividad, excepto en el último decenio. Probablemente esto sea debido a que ya estamos en un periodo de menos precipitaciones y la laguna va teniendo una tendencia a aumentar su salinidad, la cual descenderá de nuevo en el momento de las lluvias inten-

Tabla 1. Principales variables limnológicas de la Albufera de Valencia en el periodo de estudio. Datos 1975 de Del Río y Fernández (1980); 1980-81 de Serra et al. (1984). Main limnological variables of the Albufera de Valencia in the study period. Data 1975 from Del Río y Fernández (1980); 1980-81 from Serra et al. (1984).

\begin{tabular}{lccccc}
\hline Variable & 1975 & \multicolumn{2}{c}{$1980-1981$} & \multicolumn{2}{c}{$2016-2018$} \\
& (una muestra) & Media & Desv. Estándar & Media & Desv. Estándar \\
\hline Temperatura $\left({ }^{\circ} \mathrm{C}\right)$ & 15.0 & 20.8 & 6.0 & 18.9 & 6.3 \\
Conductividad $(\mu \mathrm{S} / \mathrm{cm})$ & 1527 & 1400 & 233 & 2042 & 407 \\
$\mathrm{pH}$ & 9.95 & 9.17 & 0.77 & 8.76 & 0.83 \\
Cloruros $(\mathrm{mM})$ & 8.76 & 5.66 & 1.05 & 7.70 & 2.83 \\
P total $(\mu \mathrm{M})$ & 7.4 & 15.3 & 5.1 & 3.5 & 1.9 \\
Clorofila $a(\mu \mathrm{g} / \mathrm{l})$ & Sin datos & 485.6 & 198.2 & 95.0 & 56.9 \\
\hline
\end{tabular}




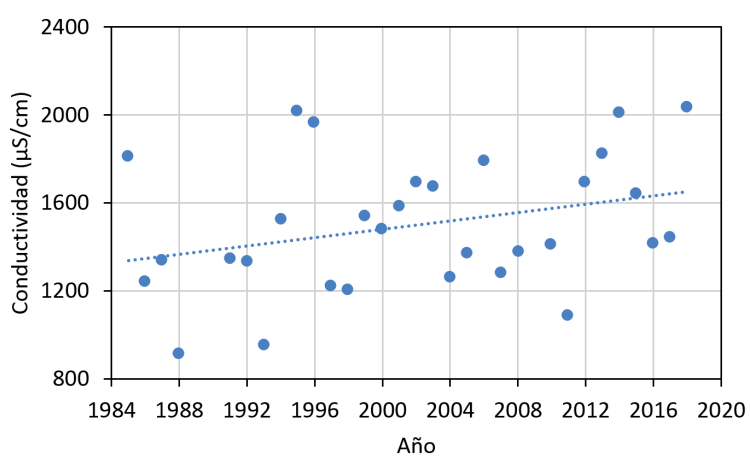

Figura 5. Tendencia del valor mínimo de conductividad de la Albufera de Valencia desde 1985 a 2018 (Mann-Kendall test, $p=0.0007$ ); la línea discontinua indica el valor de la regresión. Trend of the minimum conductivity value from 1985 to 2018 in Albufera de Valencia (Mann-Kendall test, $\mathrm{p}=0.0007$ ). The dashed line indicates the adjusted value of the series.

sas. Pero debemos tener en cuenta que el valor mínimo de la conductividad sí va aumentando con el paso del tiempo, lo cual es una señal inequívoca de que hay una tendencia a que las lluvias y las aportaciones de escorrentías superficiales, que son las que disminuyen la conductividad, sean cada vez más escasas.

Esta conductividad propia de aguas superficiales de la Albufera de Valencia la hace muy diferente a la de otras albuferas existentes en la Península Ibérica, como el Mar Menor en Murcia o las lagunas del Delta del Ebro. En la laguna murciana se da la paradoja que su comunicación con el Mediterráneo produzca una circulación estuarina negativa y una concentración hipersalina, normalmente en torno a $45 \mathrm{~g} / \mathrm{L}$, con variaciones debidas a las aportaciones de escorrentías superficiales (Peñalver et al., 2015). En las lagunas del Delta del Ebro la variación cubre todo el rango durante el ciclo anual, desde las aguas oligohalinas a las hipersalinas; es frecuente observar en ellas rangos de salinidad en un ciclo anual entre 1 y $40 \mathrm{~g} / \mathrm{L}$ para misma laguna, reflejando así los periodos de influencia marina y los de aportaciones de aguas superficiales (Comín et al., 1987).

En la laguna costera de Burrullus (Egipto), similar a la Albufera de Valencia, la situación también es muy variable. El estudio de la salinidad a lo largo de seis años ha mostrado valores entre 0.5 y $21.7 \mathrm{~g} / \mathrm{L}$, existiendo en ella una heterogeneidad espacial, con mayor salinidad en la zona este y los valores menores en la zona oeste (Nassar \& Garib, 2014). Sin embargo, en general sus aguas suelen ser oligohalinas, salvo en años más salobres. Lo contrario sucede en la laguna costera de Açu (Brasil), donde la separación del Atlántico llega a ser tan pequeña como $50 \mathrm{~m}$ (Chagas \& Suzuki, 2005), lo cual genera valores de salinidad similares a los del agua de mar en las zonas próximas, mientras que en las más alejadas los valores son la mitad que los del agua marina, dando lugar a zonas de mezcla entre las aguas dulces de origen continental y las de influencia marina. En el caso de la laguna costera de El Yali (Chile) existe conexión marina durante una época del año y una desconexión en otras estaciones (Dussailant et al., 2009); el carácter mediterráneo del clima en esta región sudamericana y la evaporación lleva a la laguna a una situación hipersalina, a pesar de las aportaciones de agua dulce, encontrándose valores promedios similares al agua de mar, pero con valores extremos que la duplican durante la estación seca.

Las situaciones de fluctuación a lo largo del año son la norma en la distribución de la conductividad en las lagunas costeras y la razón que les proporciona sus características biológicas singulares, permitiéndoles ser hábitat de numerosas especies durante una parte del año (Sigala et al., 2012). El caso opuesto, similar al de la Albufera de Valencia, lo encontramos en la laguna costera de Peri (Brasil), situada en zona subtropical donde, a pesar de la proximidad de la costa Atlántica (unos $500 \mathrm{~m}$ ), las aportaciones de aguas pluviales son tan importantes que los valores de conductividad no sobrepasan nunca los $100 \mu \mathrm{S} / \mathrm{cm}$ (Hennemann \& Petrucio, 2011), siendo, por tanto, totalmente aguas dulces.

El estudio de tendencias de la serie temporal resulta útil como indicador climático en un escenario de cambios como el que nos encontramos. Por una parte, el valor promedio no presenta variaciones; pero el ascenso continuado de los valores mínimos es un buen indicador de que no se está aportando agua dulce de origen continental a la laguna, al igual que sucede en otras lagunas costeras. 


\section{CONCLUSIONES}

La medida de la conductividad en los ecosistemas lagunares costeros es importante para conocer su situación salina y, por ello, estudiar en qué punto se encuentra en una escala de influencia entre las aguas dulces de origen continental y la influencia que el mar tiene en su hidrodinámica.

En el caso de la Albufera de Valencia, la existencia de una serie larga de variables limnológicas del agua, así como otros estudios sedimentológicos, muestran que su origen marino está relativamente próximo y que el cambio a aguas dulces $u$ oligohalinas es reciente (apenas 300 años), debido principalmente a la transformación del territorio para el cultivo del arroz. Su conductividad se mantiene en unos valores alrededor de los $2100 \mu \mathrm{S} / \mathrm{cm}$ en el estudio de la serie temporal, aunque se observe un aumento general de los valores mínimos. La conductividad del agua está relacionada con la concentración de cloruros disueltos.

La utilización de las medidas de conductividad en diferentes ubicaciones de la Albufera de Valencia se puede emplear como un indicador de los cambios en los aportes hídricos, ya sean de origen fluvial o por precipitación. Por ello, es importante mantener las actuales redes de medida, extendiéndolas también a las zonas de entrada de agua al Parque Natural. Así se conservará la integridad de la serie de datos existente y se ampliará para futuros estudios.

\section{DATASET}

La serie temporal de datos empleada está publicada en Mendeley Data (Soria, 2020).

\section{AGRADECIMIENTOS}

Al Ayuntamiento de Valencia, Diputación de Valencia, Generalitat Valenciana, Gobierno de España por las esporádicas financiaciones recibidas en diversos proyectos y a la Catedrática María Rosa Miracle por iniciar, incentivar y realizar parte de los estudios de la serie limnológica del lago de la Albufera. Agradecemos también al personal y técnicos de las entidades gestoras del Parque Natural de la Albufera y a sus pescadores y barqueros, así como a nuestros numerosos estudiantes colaboradores de la Universitat de València, su maravillosa dedicación e inestimable ayuda para poder muestrear y recopilar estas cuatro décadas de datos. Un esfuerzo así solo puede entenderse por la inmensa pasión que nos une para la conservación del Parque Natural de la Albufera.

\section{BIBLIOGRAFÍA}

AEMET, 2019. Informe climático anual 2018. Servicios climáticos de la Agencia Estatal de Meteorología. Madrid. 77 pp. On line: $\mathrm{http}: / / \mathrm{www} \cdot a e m e t . e s$.

ASLO, 1958. The Venice System for the Classification of marine waters according to salinity. Limnology and Oceanography, 3(3): 346-347. DOI: 10.4319/lo.1958.3.3.0346

CHAGAS, G. G. \& M. S. SUZUKI. 2005. Seasonal hydrochemical variation in a tropical coastal lagoon (Açu lagoon, Brazil). Brazilian Journal of Biology, 65(4): 597-607.

COMÍN, F. A., M. MENÉNDEZ \& E. FORÉS. 1987. Salinidad y nutrientes en las lagunas costeras del Delta del Ebro. Limnetica, 3: 1-8.

DEL RÍO, J. G. \& L. H. FERNÁNDEZ. 1980. La influencia de la contaminación de la Albufera de Valencia en la población de diatomeas del lago. In: Mediterranean Coastal Pollution, 473-479. Pergamon. DOI: 10.1016/B978-008-026058-7.50039-X

DUSSAILLANT, A., P. GALDAMES \& C. L. SUN. 2009. Water level fluctuations in a coastal lagoon: El Yali Ramsar wetland, Chile. Desalination, 246: 202-214. DOI: 10.1016/j. desal.2008.03.053

GARRISON, T. 2013. Oceanography: an invitation to marine science. Cengage Learning, Boston, USA. 606 pp.

HAMMER, Ø., D. A. T. HARPER \& P. D. RYAN. 2001. PAST: Paleontological Statistics Software Package for Education and Data Analysis. Palaeontologia Electronica, 4(1): 9 pp.

HENNEMANN, M. C. \& M. M. PETRUCIO. 2011. Spatial and temporal dynamic of trophic relevant parameters in a subtropical coastal lagoon in Brazil. Environmental 
Monitoring Assessment, 181: 347-361. DOI: 10.1007/s10661-010-1833-5

JÉGOU, A \& C. SANCHIS-IBOR. 2019. The opaque lagoon. Water management and governance in l'Albufera de València wetland (Spain). Limnetica, 38(1): 503-515. DOI: 10.23818/limn.38.29

LE BARS, D., S. DRIJFHOUT \& H. de VRIES. 2017. A high-end sea level rise probabilistic projection including rapid Antarctic ice sheet mass loss. Environmental Research Letters, 12: 044013. DOI: 10.1088/1748-9326/aa6512

MARCO-BARBA, J. 2010. Ecology and geochemistry of ostracods as paleoenvironmental indicators in marginal environments: the case study of Albufera de Valencia. Universitat de València, Spain (Tesis doctoral). On line: http://hdl.handle.net/10803/39091

MARGALEF, R. \& M. MIR. 1973. Indicadors de canvis de salinitat en els sediments de l'Albufera de València. Treballs de la Societat Catalana de Biologia, 32: 111-117.

MONDRÍA, M. 2010. Infraestructuras y eutrofización en l'Albufera de València. El modelo Cabhal [Tesis doctoral no publicada]. Universitat Politècnica de València. 432 pp. DOI: 10.4995/Thesis/10251/10527

NASSAR, M. Z. A. \& S. M. GARIB. 2014. Spatial and temporal patterns of phytoplankton composition in Burullus Lagoon, Southern Mediterranean Coast, Egypt. The Egyptian Journal of Aquatic Research, 40(2): 133-142. DOI: 10.1016/j.ejar.2014.06.004

PARDO, L. 1942. La albufera de Valencia: estudio limnográfico, biológico, econóomico $y$ antropolóogico. Biología de las aguas continentales. II. Instituto Forestal de Investigaciones y Experiencias. 261 pp.

PEÑALVER, J., P. MUÑOZ, E. ROMERP, E. BARCALA \& E. M. DOLORES. 2015. Primer registro de la fase juvenil de la anguila europea Anguilla anguilla en la laguna hipersalina, Mar Menor, sureste español. Revista de biología marina y oceanografía, 50(2): 391-395.

PERIS, T. 1991. La problemática génesis del Segundo tramo de la Acequia Real del Xúquer. Investigaciones Geográficas, 9: 167-190. DOI: 10.14198/INGEO1991.09.10
PRIMO, E., P. CUÑAT, J. M. CARRASCO, M. C. BLANCO \& M. MARTINEZ. 1975. Contaminación de la Albufera de Valencia. II. Niveles de contaminación por residuos urbanos. Revista de Agroquímica y Tecnología Alimentaria, 15: 98-112.

RODRIGO, M. A., J. L. ALONSO-GUILLÉN, \& I. SOULIÉ-MÄRSCHE. 2010. Reconstruction of the former charophyte community out of the fructifications identified in Albufera de València lagoon sediments. Aquatic Botany, 92: 14-22.

ROMO, S., M. J. VILLENA, M. SAHUQUILLO, J. SORIA, M. GIMENEZ, T. ALFONSO et al. 2005. Response of a shallow Mediterranean lake to nutrient diversion: does it follow similar patterns as northern shallow lakes? Freshwater Biology, 50: 1706-1717. DOI: $10.1111 /$ j.1365-2427.2005.01432.x

ROMO, S., J. SORIA, F. FERNANDEZ, Y. OUAHID, \& A. BARÓN-SOLÁ. 2013. Water residence time and the dynamics of toxic cyanobacteria. Freshwater Biology, 58(3): 513-522. DOI: 10.1111/j.1365-2427. 2012.02734.x

ROSSELLÓ, V. M. 1972. Los ríos Júcar y Turia en la génesis de Albufera de Valencia. Cuadernos de geografía, 11: 7-25.

SERRA, M., M. R. MIRACLE \& E. VICENTE. 1984. Interrelaciones entre los principales parámetros limnológicos de la Albufera de Valencia. Limnetica, 1: 9-19.

SIGALA, K., S. REIZOPOULOU, A. BASSET $\&$ A. NICOLAIDOU. 2012. Functional diversity in three Mediterranean transitional water ecosystems. Estuarine, Coastal and Shelf Science, 110: 202-209. DOI: 10.1016/j.ecss. 2012.06.002

SORIA, J. M. 1997. Estudio limnológico de los ecosistemas acuáticos del Parc Natural de l'Albufera de Valencia. Tesis Doctoral. Universitat de València. On line: http://hdl. handle.net/10550/38259

SORIA, J. M. 2006. Past, present and future of la Albufera of Valencia Natural Park. Limnetica, 25(1-2): 135-142. DOI: 10.23818/limn.25.10

SORIA, J. 2020. Conductivity Albufera of Valencia lagoon. Mendeley Data, v1. DOI: 10.17632/z6nwhxwwnh.1 
SORIA, J. M., E. VICENTE \& M. R. MIRACLE. 2001. The influence of flash floods on the limnology of the Albufera of Valencia lagoon (Spain). Verhandlungen der Internationalen Vereingung für Limnologie, 27: 2232-2235. DOI: $10.1080 / 03680770.1998 .11901635$

SORIA, J. M. \& E. VICENTE. 2002. Estudio de las aportaciones al Parque Natural de la Albufera de Valencia. Limnetica, 21: 105-116.

SORIA, J. M., M. R. MIRACLE \& E. VICENTE. 2002. Relations between physic-chemical and biological variables in aquatic ecosystems of the Albufera Natural park (Valencia, Spain). Verhandlungen der Internationalen Vereingung für Limnologie, 28: 564-568. DOI: 10.1080/03680770.2001.11901780

SORIA, J. M., M. SAHUQUILLO \& R. MIRACLE. 2005. Relaciones entre las aportaciones a la zona regable del río Júcar y la conductividad de la Albufera de Valencia. Limnetica, 24: 155-160. DOI: 10.23818/limn.24.15

SORIA-PERPINYÁ, X., M. R. MIRACLE, J. SORIA, J. DELEGIDO \& E. VICENTE. 2019. Remote sensing application for the study of rapid flushing to remediate eutrophication in shallow lagoons (Albufera of Valen- cia). Hydrobiologia, 829(1): 125-132. DOI: 10.1007/s10750-018-3741-6

SORIA-PERPINYÁ, X., E. VICENTE, P. URREGO, M. PEREIRA-SANDOVAL, A. RUIZ-VERDÚ, J. DELEGIDO, J. M. SORIA \& J. MORENO. 2020. Remote sensing of cyanobacterial blooms in a hypertrophic lagoon (Albufera of València, Eastern Iberian Peninsula) using multitemporal Sentinel-2 images. Science of the Total Environment, 698: 134305. DOI: 10.1016/j.scitotenv.2019. 134305

VICENTE, E. \& M. R. MIRACLE. 1992. The coastal lagoon Albufera de Valencia: An ecosystem under stress. Limnetica, 8: 87-100.

YODER, L. 1919. Adaptation of the Mohr volumetric method to general determinations of Chlorine. Industrial \& Engineering Chemistry, 11: 755. DOI: 10.1021/ie50116a013

ZARIQUIEY, R. 1935. Adiciones al ensayo de un catálogo de los crustáceos decápodos de España y Marruecos, de D. Alvaro Miranda. Butlletí de la Institució Catalana d'Història Natural, 1935: 92-98, On line: https://www. raco.cat/index.php/ButlletiICHN/article/view/ 21626 [Consulta: 19-05-2020]. 